\title{
Critical Phenomena and Breaking Drops: Infinite Idealizations in Physics
}

\author{
Robert W. Batterman ${ }^{1}$ \\ Ohio State University \\ (C) 2004
}

\footnotetext{
${ }^{1}$ I would like to thank Gérard Emch, Malcolm Forster, Leo Kadanoff, Nicholaos Jones, Sid Nagel, and Sheldon Smith for helpful comments, criticism, and discussion. A version of this paper was presented at the ESF Conference on Philosophical and Foundational Issues in Statistical Physics, Utrecht 28-30 November 2003. (Special thanks to Jos Uffink and Dennis Dieks for a wonderful conference.)
} 


\section{Introduction: Intertheory Relations in Physics}

The study of intertheoretic relationships is a prime topic for philosophers of science. Typically such studies involve questions about whether one theory reduces to another, and if so, what is the best way to characterize this relationship. Most contemporary workers in this area pay homage to Ernest Nagel's [15, Chapter 11] seminal discussion of theory reduction. In the crudest form, Nagel and his followers hold that a typically older and coarser theory $T_{c}$ reduces to a typically newer and more refined theory $T_{f}$ according to the following scheme:

(N) $T_{c}$ reduces to $T_{f}$ just in case the laws of $T_{c}$ are derivable from those of $T_{f}$.

As an example, we can think of $T_{f}$ as special relativity and $T_{c}$ as the Newtonian theory of space and time. Thus the Newtonian theory reduces to the special relativistic theory, because some kind of derivational relationship obtains between the laws of the two theories. Much work over the years has been devoted to refining and elaborating this schema. (Nagel, himself, said nothing remotely so crude.) Nevertheless, for our purposes here, I think that this is sufficient to begin with. ${ }^{1}$

In contrast to this "philosopher's conception" of reduction, is what I call a "physicist's conception." This view was first noted and discussed by Thomas Nickles [17] in paper entitled "Two Concepts of Intertheoretic Reduction." On this view the finer theory reduces to the coarser theory in some appropriate limit.

$$
\lim _{\epsilon \rightarrow 0} T_{f}=T_{c} .
$$

Thus, the special theory of relativity reduces to the Newtonian theory of space and time in the limit in which the relative velocities of the reference frames are small compared with the speed of light.

Much of my recent work has been focused on the schema (1). ${ }^{2}$ In particular, I have argued that one cannot have interesting philosopher's reductions, without a physicist's reduction obtaining. Furthermore, and this is the crucial point for the current discussion, for most theory pairs $T_{f}$ and $T_{c}$, the

\footnotetext{
${ }^{1}$ On the other hand, we will shortly have to become a bit more sophisticated in our understanding of this "philosopher's conception" of reduction.

${ }^{2}$ In particular, see [1].
} 
physicist's schema actually fails. Thus, most cases of purported reductions between theories will actually fail to be reductions at all. The reason for this is really quite simple. The equality in (1) can obtain only if the limit is regular. If the solutions of the relevant formulas or equations of the theory $T_{f}$ are such that for small values of $\epsilon$ they smoothly approach the solutions of the corresponding formulas in $T_{c}$, then the limit is regular and (1) will be satisfied. For such cases we can say that the "limiting behavior" as $\epsilon \rightarrow 0$ equals the "behavior in the limit" where $\epsilon=0$. On the other hand, if the behavior in the limit is of a fundamentally different character than the nearby solutions one obtains as $\epsilon \rightarrow 0$, then the limit is singular and the schema will fail.

Examples where the physicist's schema fails include the short wavelength $(\lambda \rightarrow 0)$ limit of the wave theory of light and the semiclassical $(\hbar \rightarrow 0)$ limit of quantum mechanics. These limits both fail to approach smoothly the solutions to the coarser theories - ray optics and classical mechanics, respectively.

Another limiting relationship that fails to be regular is the topic of this paper - the thermodynamic limit of statistical mechanics at critical points. The situation here is very subtle indeed and the study of the limiting failures bring much of interest physically, mathematically, and philosophically.

Here, I want to investigate further the consequences of the singular nature of schema (1) for understanding the relationships between statistical mechanics and thermodynamics. One point I would like to make is that a failure to realize the import of this singular relationship has recently led to a mistaken view about the role of the thermodynamic limit and the idealizations according to which statistical mechanics must treat systems as containing an infinite number of particles. In particular, I intend to take issue with Craig Callender's claim in "Taking Thermodynamics Too Seriously" [3] that such an idealization leads to fundamental mistakes. On the contrary, once we understand that reductive relations between thermodynamics and statistical mechanics completely fail at critical points and phase transitions, we will see that the infinite idealizations are completely justified.

The conclusion generalizes. I will consider, by way of further supporting the legitimacy of limiting infinite idealizations, a second example. This is the everyday phenomenon in which fluid such as water from a dripping faucet undergoes a singular evolution as it breaks apart into drops. Despite obvious differences, the two cases are, in fact, quite similar. 


\section{The "Received" View}

In order for thermodynamics to reduce to statistical mechanics in the philosopher's sense of schema $(\mathrm{N})$, one must be able to derive the thermodynamic behavior of a system as captured by the laws of that theory from the statistical mechanical laws that take seriously the idea that such a (thermal) system is composed of a large number of particles. Quite famously, primarily because of Nagel's presentation, the reduction of thermodynamics to statistical mechanics is taken to be the paradigm instance of theory reduction in the physical sciences. However, as recent work has shown, this is a mistake. ${ }^{3}$ Many problems arise including how one can identify the nonstatistical concepts appearing in thermodynamics with the statistical/ensemble quantities that play such a crucial role in statistical mechanics. Such identifications or bridge laws are necessary for the derivation demanded by schema $(\mathrm{N})$, nevertheless, their validity, to say the least, is not at all straightforward.

More serious is the fact that at critical points, it seems that there can be no schema $(\mathrm{N})$-type derivation whatsoever, even if we were able to solve the various problems about bridge laws. This is because the physicist's schema, (1) is singular at those locations in phase. I will say more about this below. For now note that one way in which the singular nature of this limit manifests itself is in the failure of so-called Mean Field Theory to accurately represent what goes on at criticality. Let me now introduce these terms and discuss an important argument leading to what I take to be the received view of the relationship between thermodynamics and statistical mechanics at critical points.

Consider a magnet modeled as a system of spins on a lattice. The magnetization of the system (in the absence of an external magnetic field) is produced by the spontaneous alignment of the spins. Such states, again in the absence of an external field, are degenerate - for every state in which the average magnetization $m$ of the spins points some direction $\sigma$ there is a thermodynamically equivalent state in which it points in the opposite direction. An important quantity for understanding the critical behavior of a system is the so-called "order parameter" which for the magnet is defined to be average magnetization $m=m(h, T)$ where $h$ is the magnetic field and $T$ is the temperature. As we see in figure 1, below the critical temperature $T_{c}$ there are two values of the magnetization for every temperature $T$, whereas

\footnotetext{
${ }^{3}$ See Sklar's Physics and Chance [21] for a discussion.
} 
above $T_{c}$ the magnetization is zero. Therefore, at zero field $(h=0)$ there is a first order phase transition for $T<T_{c}$ and as $T \rightarrow T_{c}$ from below that discontinuity vanishes indicating the existence of a second order (or continuous) phase transition at the critical point.

It is well-known that so-called Mean Field Theory gives a qualitatively correct characterization of phase transitions in magnetic systems. This theory employs the assumption that each spin acts as if it is independent of the others feeling only the average (mean field) produced by all of the other spins. In making this assumption, Mean Field Theory explicitly ignores fluctuations in the ordering of the spins on the lattice. This has the unfortunate consequence that Mean Field Theory completely fails to reproduce the experimentally observed critical exponent $\beta$ that appears in the scaling law for the magnetization:

$$
m \propto|t|^{\beta}
$$

where

$$
t=\frac{T-T_{c}}{T_{c}}
$$

is the reduced temperature describing the deviation of the system from criticality in dimensionless units. The Mean Field prediction for $\beta$ is $1 / 2$, whereas a value much closer to .325 is actually observed.

Mean Field Theory, because it ignores fluctuations, also ignores the fact that near criticality the correlation length-roughly, the distance over which significant spin correlations occur-becomes extremely large and actually diverges at the critical point. In fact, though I won't rehearse the argument here, it is possible to use Mean Field Theory itself to estimate the importance of such fluctuations. As a result, the theory, in effect, predicts its own failure. The argument is due to V. L. Ginzburg [10]. See [13, pp. 242-243] and [11, pp. 169-172] for discussions.

In statistical mechanics every thermodynamic function can be determined from the partition function $Z$ which is related to the free energy $F$ through the relation:

$$
Z(\beta)=e^{-\beta(F(\beta, \lambda)},
$$

where $\lambda$ is a some parameter appearing in the system's Hamiltonian and $\beta$ is the inverse temperature $-\beta=1 / k T{ }^{4}$ In particular, the magnetization $m$

\footnotetext{
${ }^{4}$ It is an unfortunate convention that $\beta$ is also used to represent the exponent in the scaling law for the order parameter $m$. Context should make clear to what it refers.
} 


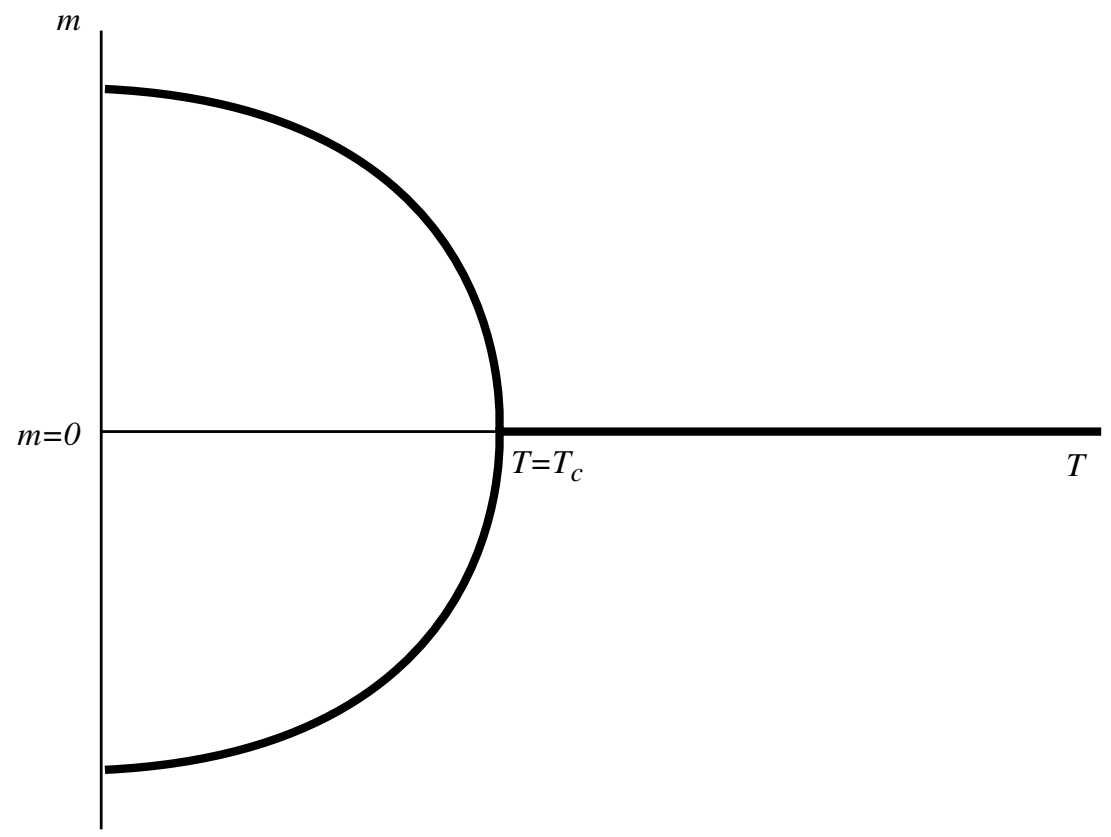

Figure 1: Magnetization vs. Temperature at zero Field 
is determined as follows:

$$
m=\frac{\partial}{\partial h} \ln Z
$$

For our system of $N$ spins on a lattice, the partition function is a sum of $2 N$ terms representing the different possible configurations of the spins. Each such term is a positive exponential and there is a mathematical result stating that a finite sum of positive exponentials must be an analytic function. It is, therefore, impossible for the magnetization to be a nonanalytic function. Phase transitions and, in particular, the continuous phase transition at the critical point, are represented in thermodynamics as nonanalyticities in the relevant thermodynamic potentials. This leads immediately to the conclusion that for a system with a finite number of particles or degrees of freedom, there can be no phase transitions. As a result, no derivation of the critical behavior described by thermodynamics from statistical mechanical considerations is possible. The philosopher's reduction fails.

On the other hand, Kadanoff [13, p. 238] holds that this argumentsuggesting as it does that nonanalyticities are impossible -is "spurious". He believes that the theorem is inapplicable since it is possible for nonanalytic behavior to appear in systems of infinite size - that is, with infinite $N$. He says that from this argument we must conclude that

The existence of a phase transition requires an infinite system. No phase transitions occur in systems with a finite number of degrees of freedom. [13, p. 238]

Mean Field Theory for infinite $N$ provides a qualitative description of phase transitions and critical phenomena in the thermodynamic limit. Quantitatively, it is accurate away from critical points but fails at criticality in that (among other things) it predicts the incorrect value of $1 / 2$ for the exponent $\beta$. The idealization of infinite $N$ - the thermodynamic limit - is necessary for the qualitative characterization of phase transitions and critical phenomena as Kadanoff's argument demonstrates, but it is insufficient for an accurate quantitative description of what goes on at criticality.

Now, I think that it is fair to say that the view of phase transitions and critical phenomena as requiring the limit of infinite $N$ is the dominant view among physicists. ${ }^{5}$ However, a little reflection suggests that this dominant or "received" view is not quite so straightforward. When pressed with the fact

\footnotetext{
${ }^{5}$ There are dissenters, however. See, e.g., [12].
} 
that actual physical systems are composed of a finite number of particles, most people respond by saying something along the following lines: "Well, the thermodynamic limit is really an idealization, what we mean is that actual systems approximate in some way this idealized limiting case." 6 In other words, they hold that from a purely mathematical perspective, we do need the thermodynamic limit. But in the real world of physics, our appeal to such a limiting idealization is really a pragmatic move that will help us deal with very messy detailed calculations.

Such a response is clearly at odds with Kadanoff's conclusion that infinite systems are necessary for phase transitions. I think that this "elaborated received view", which endorses Kadanoff's conclusion while simultaneously appealing to the pragmatic need to approximate, is an unstable position. On the one hand, perhaps we should take seriously the fact that real systems are finite and search for an account of phase transitions and critical phenomena in terms of the statistical mechanical partition function for systems with finite $N$. On the other hand, if we do this, we run afoul of the theorem mentioned above to the effect that phase transitions for finite $N$ systems are impossible.

In the next section I will consider an argument to the effect that we ought to look for an account of phase transitions that takes seriously the fact of finite $N$ and dismisses the relevance of the theorem.

\section{Taking Thermodynamics Too Seriously?}

Craig Callender [3], I think, can be understood as reasoning to this effect. He argues that when we assert that the thermodynamic limit is essential for characterizing phase transitions and critical phenomena, we are "taking thermodynamics too seriously" - that is, we are taking the thermodynamic representation of phase transitions and critical phenomena as nonanalytic behaviors in the various thermodynamic potentials too literally.

He recognizes the mathematical theorem we have been discussing:

The problem is that phase transitions - as understood by statistical mechanics - can only occur in infinite systems, yet the phenomena that we are trying to explain clearly occur in finite systems. [3, p. 549]

\footnotetext{
${ }^{6} \mathrm{I}$ am not saying that this is Kadanoff's view of the matter.
} 
Further, after allowing that from a pragmatic point of view the statistical mechanical idealization of real systems as having an infinite number of molecules is a very good thing - such an idealization enables us to ignore boundary effects, it enables us to minimize (in fact eliminate fluctuations), etc.-Callender still insists that there is something philosophically mistaken about such an idealization. He says,

$[\mathrm{o}] \mathrm{n}$ the other hand, the thermodynamic limit may not be a good approximation in a more foundational, philosophical sense. ... [E]ven if the thermodynamic limit can be given a full philosophical justification, that justification cannot turn an infinity into a finite quantity. We can grant that it is often fine to substitute finite $N$ with infinite $N$ for purposes of practical physics. But if the system is really finite $N$, what we have until we say more is a mathematical proof that it cannot undergo a phase transition. So we ought to grant that, practically speaking, it is often a good approximation, but point out that this just does not touch this particular problem. ...

$[\mathrm{T}]$ he fact that thermodynamics treats phase transitions as singularities does not imply that statistical mechanics must too. To assume that would be to take thermodynamics too seriously. ... Thermodynamics represents (for pretty good reasons) phase transitions as singularities, and statistical mechanics (for pretty good pragmatic reasons) takes this to mean a non-analyticity in the partition function. But from a foundational perspective we cannot endorse this knee-jerk identification of mathematical definitions across levels. [3, p. 550]

We take thermodynamics too seriously when (from the point of view of statistical mechanics) we take seriously the thermodynamic conception of a phase transition as mathematically represented by a discontinuity or singularity in a thermodynamic potential such as the free energy $F$. Thus, this must mean that such a mathematical representation is somehow to be faulted. In other words, the equations of thermodynamics must be physically inadequate. Hence, the inadequacy of the thermodynamic equations is ultimately responsible for our (apparently) mistaken conviction that the thermodynamic limit is required to "derive" phase transitions from fundamental statistical mechanics. To the contrary, Callender insists that analytic 
partition functions must govern the phase transitions we see in real systems and must "in some sense approximate a singularity." [3, p. 555]

I will argue below that this view is inadequate. Thus, I want to champion the manifestly outlandish proposal that despite the fact that real systems are finite, our understanding of them and their behavior requires, in a very strong sense, the idealization of infinite systems and the thermodynamic limit. Before getting to this, let me try to provide another perspective on why one might think that appeal to the thermodynamic limit and infinite $N$ is a mistake - why, that is, that we might be taking thermodynamics too seriously.

\subsection{A More Sophisticated Attempt at Reduction}

As I mentioned earlier, schema $(\mathrm{N})$ - the philosopher's view of reduction - is too crude as it stands. ${ }^{7}$ Often in attempting to reduce a theory $T_{c}$ to $T_{f}$ in the philosopher's sense we find that we need to amend or correct the reduced theory $T_{c}{ }^{8}$ Most physicists, now, would accept the idea that our concept of temperature and our conception of other "exact" terms that appear in classical thermodynamics such as "entropy", need to be modified in light of the alleged reduction to statistical mechanics. Textbooks, in fact, typically speak of the theory of "statistical thermodynamics" allowing explicitly for fluctuations that one observes in thermodynamic systems. The very process of "reduction" often leads to a corrected version of the reduced theory.

Schaffner [18, p. 618], for instance, has developed a relatively sophisticated version of the Nagelian reduction strategy that explicitly takes this fact into consideration. Let us call this schema (NN), for "neoNagelian".

(NN) $T_{c}$ reduces to $T_{f}$ if and only if there is a corrected version of $T_{c}$ (call it $T_{c}^{*}$ ) such that

1. The primitive terms of $T_{c}^{*}$ are associated via reduction functions (or bridge laws) with various terms of $T_{f}$.

2. $T_{c}^{*}$ is derivable from $T_{f}$ when it is supplemented with the reduction functions specified in 1 .

3. $T_{c}^{*}$ corrects $T_{c}$ in that it makes more accurate predictions than does $T_{c}$.

\footnotetext{
${ }^{7}$ Again, Nagel, himself, would take $(\mathrm{N})$ to be a caricature of the position.

${ }^{8}$ See [20] and [18].
} 
4. $T_{c}$ is explained by $T_{f}$ in that $T_{c}$ and $T_{c}^{*}$ are strongly analogous to one another, and $T_{f}$ indicates why $T_{c}$ works as well as it does in its domain of validity.

Here the idea is that we can have a reduction of thermodynamics to statistical mechanics if there is a corrected version of the orthodox thermodynamic theory - statistical thermodynamics - that plays the role of $T_{c}^{*}$ in schema (NN). The real work to be done is in spelling out what "strongly analogous" means in clause 4 . One way of attempting to elucidate this notion is by appeal to the existence of a limiting relationship between statistical thermodynamics and the orthodox theory along the lines suggested by schema (1).

On the assumption that the relationship between thermodynamics and statistical mechanics fits this sophisticated neoNagelian reduction program, we can see why we would be taking thermodynamics too seriously when we need to appeal to the thermodynamic limit of statistical mechanics to explain/reduce the thermodynamic theory. In other words, perhaps we can account for the "apparent" necessity of the infinite $N$ idealization by noting that we have failed to realize that orthodox thermodynamics too literally treats phase transitions as real discontinuities. Once we see that the theory is really corrected in the process of reduction, this literal reading will be seen to be too strong. Callender actually suggests something like this in a different context: He holds that the statistical mechanical identification of a stationary probability distribution with thermal equilibrium results from a failure to realize that (orthodox) thermodynamics too literally treats equilibrium states as completely constant and fluctuation free. ${ }^{9}$ [3, pp. 545-547] Statistical thermodynamics $\left(T_{c}^{*}\right)$ clearly does not make this mistake.

So on the current proposal, the idealization of infinite $N$ is misguided, at least in part because the reduced theory - orthodox thermodynamics - needs to be corrected and not read at face value. Had theorists a more sophisticated, less naive, view of reduction, they would not have taken thermodynamics so seriously and they would not have made this mistake. Despite this argument, I still believe that the conclusion that the infinite idealization is unwarranted, is mistaken. In the next section I begin to argue for this claim.

\footnotetext{
${ }^{9}$ Callender thinks that the identification of such Gibbsian ensembles with thermodynamical equilibrium states is a mistake. I take it that his reasons for this can be seen as resting upon an endorsement of an understanding of reduction as involving the correction of the reduced (thermodynamic) theory according to something like schema (NN).
} 


\section{Taking Thermodynamics Seriously}

Let me begin by making a distinction between two types of discontinuities, broadly construed - "physical discontinuities" and "mathematical discontinuities." As an instance of a physical discontinuity we can take the phenomenon with which we are concerned, namely, the observed qualitative distinctions between the distinct phases of a magnet (above and below the critical point) or the distinctions between the phases of a "fluid" - gaseous, liquid, and solid. Another example of a physical discontinuity, to be discussed in detail in the next section, is the obvious discontinuity that obtains when a droplet breaks off from a stream of water coming out of a faucet.

These physical discontinuities are represented mathematically as blowups or singularities in the equations that purportedly describe the behaviors of the various systems. In the former these are mathematical singularities (kinks in the derivatives of the thermodynamic potentials) in the (static or quasistatic) thermodynamic equations. In the latter case, the phenomenon is dynamical and the mathematical discontinuities are singularities in the Navier-Stokes equations of fluid dynamics - infinities that develop in finite time in the evolution of the fluid as it drips from a faucet.

The real question is whether the singularities in the mathematical representations (the equations) have genuine physical significance. Callender, as I understand him, answers this question in the negative. He agrees with Liu [14] that "[a]ctual systems are finite and phase transitions in them are never real singularities." [3, p. 550, note] I take it, given my distinction above, that this means (i) there are no physical discontinuities and (ii) the mathematical discontinuities are, therefore, nonrepresentative.

Now, perhaps, one might argue that the qualitative distinctions between phases of a magnet or of a fluid system are not genuine physical discontinuities. I don't really see how the argument might go other than to appeal to the fact that the equations are mathematically singular and this requires, as we have seen, the "unrealistic" idealization of the thermodynamic limit. But, if the distinction between physical and mathematical discontinuities makes sense, this is to beg the question. Besides, it surely does seem very plausible to describe the breakup of water into droplets as a genuine physical discontinuity. It is true that we do not see the topological change in the phase transition (say when we witness water boiling in a tea kettle) in the same way we see a stream of fluid break apart. But that, by itself, does not show that there is no genuine physical discontinuity in the thermodynamic system. 
My contention is that thermodynamics is correct to characterize phase transitions as real physical discontinuities and it is correct to represent them mathematically as singularities. Further, without the thermodynamic limit, statistical mechanics would completely fail to capture a genuine feature of the world. Without the thermodynamic limit, in fact, statistical mechanics is incapable even of establishing the existence of distinct phases of systems.

If one takes the view that there are no real physical discontinuities (recall Callender's endorsement of Liu's claim that "phase transitions [in finite systems] are never real singularities"), then I suppose there is no reason for statistical mechanics to employ the thermodynamic limit. But if there are genuine physical discontinuities and it is the goal of statistical mechanics to describe them and to explain how they are possible, then this move simply will not work. Below in section $5 \mathrm{I}$ will discuss the case of breaking droplets. The aim there will be, in part, to provide further evidence that real physical discontinuities do exist, and require infinite idealizations for their explanation and understanding. For now, let me continue to examine the thermodynamic situation on the assumption that critical phenomena are real physical discontinuities.

One might think of Mean Field Theory as an attempt to realize Schaffner's more sophisticated version $(\mathrm{NN})$ of the philosopher's reductive derivation of thermodynamic behavior from statistical mechanical assumptions. As Mean Field Theory involves the assumption that a given spin on a lattice behaves as if it were independent of its neighbors and feels only the average field produced by all the other spins, it cannot truly be a realization of the reductive program, since it involves a rather fundamental idealization about the reducing theory. However, Mean Field Theory is quite accurate in reproducing the noncritical behavior of thermodynamic systems and it does, in fact, provide qualitative accounts of phase transitions. It succeeds in this, in part, because it employs the limit of infinite $N$.

Now, Callender appeals to Mean Field Theory in support of our "faith" in the view that statistical mechanics can proceed without the thermodynamic limit. He notes that "[w]e have mean field theory, which is very accurate except near some critical points." [3, p. 551] This is true, but Mean Field Theory does employ the thermodynamic limit! So how, exactly can Callender appeal to Mean Field Theory as a (partial) justification for our belief that we can derive approximations to (orthodox) thermodynamic singularities? Now, I do not think that Callender (or any sane person for that matter) would want to eliminate all idealizations in physics - that would be like claiming 
that we could not speak of frictionless planes, etc. And fact, away from those pesky critical points the limit of infinite $N$ is relatively unproblematic. It is similar in certain respects to the idealization of frictionless planes. For instance, we can explain fluctuations appearing in actual finite systems, in a way analogous to being able to deal with the introduction of a bit of friction that real inclined planes possess. We can make precise predictions about the extent of fluctuations in large but finite systems away from criticality despite (in fact, because of) our use of the infinite $N$ idealization. And, we may be able to say in what sense our finite systems actually approximate a singularity at first order phase transitions.

One way of expressing this is to say that the idealization of infinite $N$ is controllable in those contexts - away from critical points. The idea that some idealizations are controllable and others uncontrollable is prevalent in physics. However it is a difficult task indeed to try to make the distinction precise. Very roughly, let us say that an idealization is controllable means that it is possible, via appeal to theory, to compensate in some way for the idealization. See Sklar [22] for an extended discussion of this distinction.

Thus, in some sense, Mean Field Theory enables one to provide something like a physicist's reduction of statistical mechanics to (corrected) thermodynamics according to schema (1) at least for systems not at criticality. As a result, away from critical points we may very well expect to satisfy, with various caveats, the more sophisticated philosopher's model of reduction as well.

Nevertheless, as we have seen above, and as Callender himself acknowledges, Mean Field Theory fails to characterize correctly the detailed thermodynamics of critical systems. That theory is completely incapable of dealing with the fluctuations that arise in the neighborhood of a critical point. At critical points it doesn't even let us talk about approximation in the relevant sense. The reason is that at critical points the limit $N \rightarrow \infty$ is uncontrollable - that is, it is singular and the behavior as $N \rightarrow \infty$ is qualitatively distinct from the behavior at $N=\infty$. We may get numerical results for large finite $N$ that "look close" to the nonanalytic results for infinite $N$. But "closeness" in this sense is not relevant: Smooth curves are smooth no matter how sharp they appear. And, if there exist genuine physical discontinuities, then their faithful mathematical representation demands curves with kinks. A sense of "approximation" that appeals to how similar the smooth curves are to the kinky curves is inapposite.

Let me summarize the discussion of this section. As Callender notes, 
"Thermodynamics represents (for pretty good reasons) phase transitions as singularities, and statistical mechanics (for pretty good pragmatic reasons) takes this to mean a non-analyticity in the partition function." [3, p. 550] Thermodynamics represents phase transitions and critical phenomena as singularities for very good reasons indeed. It does so because real systems exhibit physical discontinuities. As a result, I think we should take thermodynamics very seriously on this point. We should not fault thermodynamics for representing such discontinuities as singularities.

One might diagnose the failure of the naive derivational reduction as the result of taking (orthodox) thermodynamics too seriously. Instead, we should look for some corrected account whereby we can find analytic partition functions governing phase transitions that, as Callender says, "in some sense approximate a singularity." [3, p. 550]

But the failure of schema (1) at criticality indicates that even this neoNagelian reduction strategy must fail. The limit is singular and, as a result, no such corrected, approximate account is possible. This manifests itself in the failure of Mean Field Theory to correctly produce the exponent $\beta$ in equation (2). The idea that we can find analytic partition functions that "approximate" singularities is mistaken, because the very notion of approximation required fails to make sense when the limit is singular. The behavior at the limit (the physical discontinuity, the phase transition) is qualitatively different from the limiting behavior as that limit is approached.

As we know, it is possible to provide a mathematical proof that for any finite $N$ the partition function $Z$ is analytic. The limit $N \rightarrow \infty$, therefore, is a necessary condition for obtaining mathematical discontinuity in $Z$. But the failure of that limit to be regular tells us further that Mean Field Theory even in the thermodynamic limit cannot correctly represent the real physical discontinuities that are accurately captured by the thermodynamic equations. New statistical mechanical techniques (renormalization group theory) are required to deal with the singularities in the thermodynamic limit at critical points. In particular, the renormalization group techniques take advantage of the fact that at criticality, there is no characteristic length scale. These techniques, in effect, exploit this fact about the singularity to develop scaling or similarity solutions that can then be employed to explain the observed universal behavior. I won't go into the details here, but they are similar (no pun intended) to the techniques to be discussed in the next section. Furthermore, it is no accident that the techniques are related to one another. The problems of dealing with singularities in these equations are quite analogous. 
As a consequence of the singular nature of the thermodynamic limit at critical points, I believe that one ought to give up on the goal of reducing one theory to the other. The singular nature of the thermodynamic limit suggests that much of interest remains to be investigated by examining the details of the singular asymptotic domain. It therefore, suggests that the idea of infinite $N$ is not so readily dismissed as Callender and others would like it to be.

To be more precise, the worry about the infinite $N$ idealization per se is not the real issue. In some cases that idealization is controllable and provides no real interpretive problems. The real issue is the existence of genuine physical discontinuities - real singularities in the physical system. One needs mathematics that will enable one to represent these genuine physical discontinuities. As a result, there is something deeply right about the thermodynamic representation of the singularities - something that needs to be taken very seriously. Callender is right to argue that a "knee-jerk" identification of singularities between the theories should be avoided. But, the fact that the limit is singular at critical points is really an indication that the idealization cannot be dismissed so readily.

The next section discusses the other example mentioned earlier. This concerns understanding of the structure of breaking droplets. Here too we will see that there is something deeply correct about the "unrealistic" idealization that accompanies the hydrodynamical equations required for the representation of real singularities. This is the idealization that we are dealing with a continuum, as opposed to, once again, a finite collection of molecules governed by molecular dynamical equations.

\section{$5 \quad$ Breaking Droplets}

The problem of describing and explaining the nature of drop formation in fluids received its first systematic investigation in the $19^{\text {th }}$ century. Despite this, it is fair to say that real progress on this problem has been achieved only within the last 40 years or so with much work being accomplished in the last decade. One reason for this is the recognition that various linear theories were inadequate, and that a full theory would involve the investigation of the nonlinear Navier-Stokes equations for free surface flows. ${ }^{10}$

\footnotetext{
${ }^{10}$ See [6] for a recent review.
} 
As water drips from a faucet it undergoes a topological change - a single mass of water changes into two or more droplets. This is perhaps the most common example of a hydrodynamical discontinuity that arises in a finite period of time. It was recognized early by Lord Rayleigh that drops form as a result of a competition between gravitational force and surface tension. He was able to determine the typical size of a droplet and was able to set the time scale upon which a drop would form. [6, p. 866]

Contemporary work has focused on the shape of the fluid interface near the time of breakup. As the interface breaks, the thickness of the fluid must become very small. In fact, it must necessarily become smaller than any other macro-length scale that figures in the problem, such as the nozzle diameter. This has the consequence that the fluid thickness is essentially the only length (prior to reaching atomic scales) that can effect the shape of the fluid near the singularity or breakup. In particular, it is natural to assume that the shape of the fluid at different times near the breakup will differ only by a change in scale. In such a situation it is reasonable to look for a similarity solution describing the radius of the drop as a function of the vertical length $z^{\prime}$ and the time $t^{\prime}$ both measured from the point of breakup at $z_{0}$ and $t_{0}\left(z^{\prime}=z-z_{0}\right.$ and $\left.t^{\prime}=t-t_{0}\right)$. (See figure 2.) Such a solution has the form

$$
h\left(z^{\prime}, t^{\prime}\right)=f\left(t^{\prime}\right)^{\alpha} H(\zeta)
$$

where

$$
\zeta=\frac{z^{\prime}}{f\left(t^{\prime}\right)^{\beta}} \cdot{ }^{11}
$$

Eggers and Dupont [8] argued that a similarity solution of this form does indeed hold for the problem at hand. In fact they argue that such a solution is in good agreement with solutions to the Navier-Stokes equation (or approximations thereof) for low viscosities. ${ }^{12}$

This similarity solution must break down as the radius of the fluid neck reaches atomic scales. There, surely, microscopic structure becomes relevant

\footnotetext{
${ }^{11}$ The scaling exponents $\alpha$ and $\beta$ are constants to be determined, as are the functions $f$ and $H$. Note also, that $\beta$, here, has not special meaning and is is different from both its occurrences earlier on in the paper.

${ }^{12}$ Shi, Brenner, and Nagel [19] argue that Eggers' and Dupont's solution needs to be corrected as there are perturbations (noise) that play an essential role in determining the character of the fluid shape near breakup.
} 


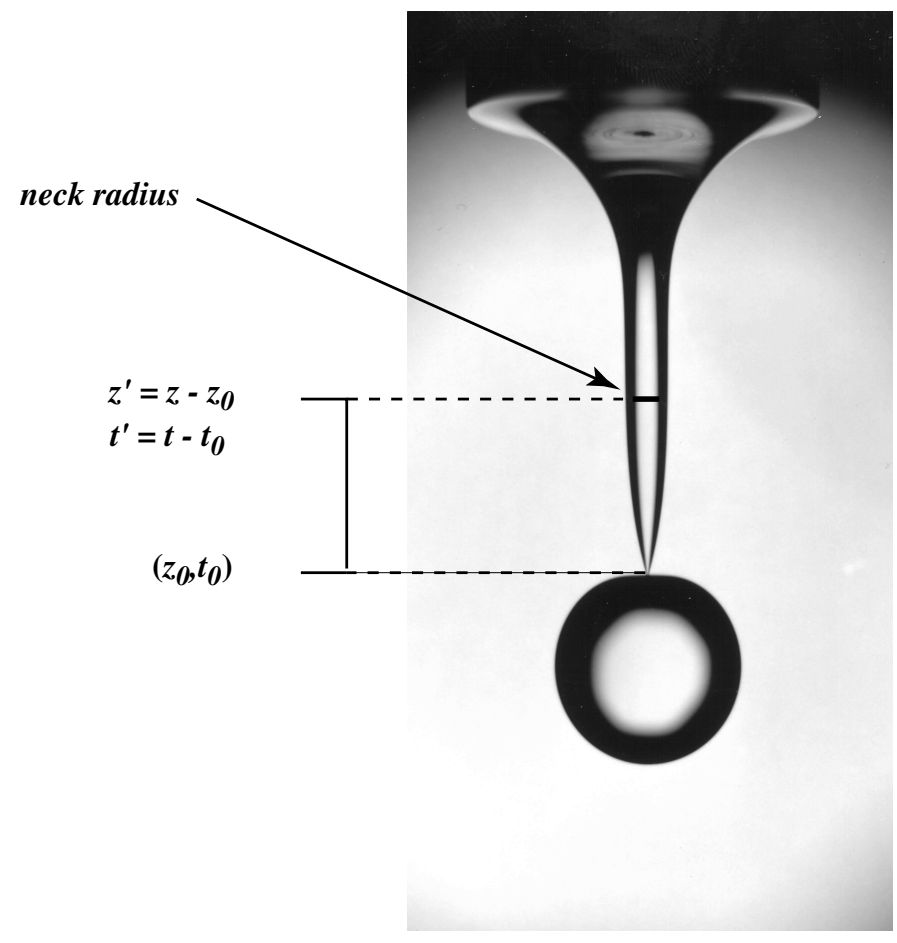

Figure 2: Neck Radius $h$ vs. $z$

for a complete description of the breakup. The analogy between this and the thermodynamic problematic discussed in the last section is clear: How can a continuum theory provide a realistic representation of the breakup when ultimately (from a "foundational, philosophical" perspective) the particulate makeup of the system plays a role in its behavior? From such a (microscopic) perspective there can be no discontinuity just as there can be no real singularity at criticality from the point of view of a theory that takes seriously the fact that statistical systems have finite $N$. Perhaps we take the hydrodynamic theory too seriously when we use it to represent the breakup of the fluid neck into droplets. Perhaps we need to include a microscopic length scale in the full description of the droplet problem.

Following the breakup, the new surfaces that appear (on the neck side and on the droplet side) are again governed by similarity solutions to the Navier-Stokes equation. So an important question is whether we need to include a microscopic length scale in our attempt to find a continuation of 


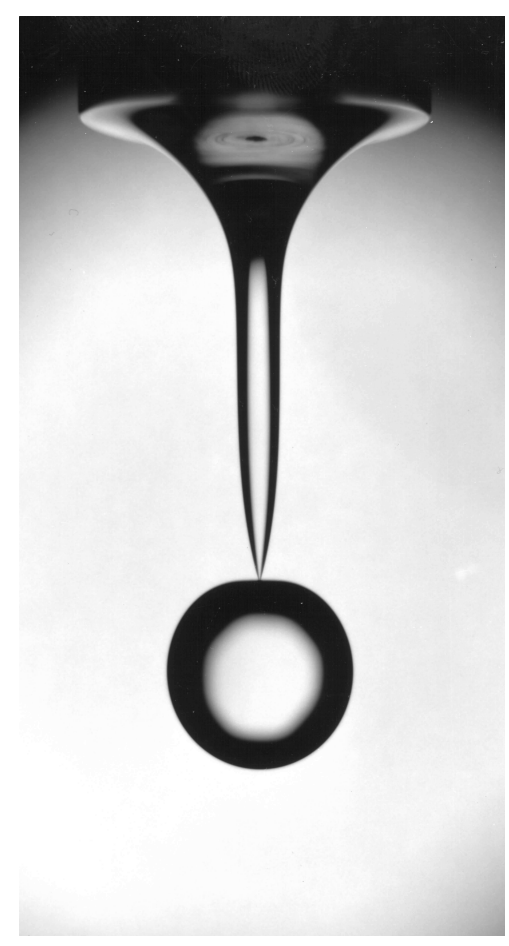

Figure 3: Water Droplet at Breakup

the Navier-Stokes equation from before breakup to after breakup - that is, across the singularity. If such a scale were required, then the hydrodynamic description would not be self-consistent, and we would have reason to suspect that we were taking that theory too seriously.

However, Eggers shows, for scales sufficiently greater than the microscopic, that it is possible to continue the solution for the before-the-breakup fluid to that for the after-the-breakup formation of drops and satellite droplets. [5] [6, pp. 894-904] Thus, the hydrodynamic theory of the breaking - the topological transition - is self-consistent and "the dynamics very quickly "forget' the microscopic details of breaking." [7, p.306] As long as we are interested in scales larger than the microscopic, the hydrodynamic description is completely adequate.

A couple of caveats are in order here: First, it is true (as noted in footnote 12) that perturbations disturb the Eggers-Dupont similarity solution. Nevertheless, as Brenner, et al. [2] suggest, thermal fluctuations producing 


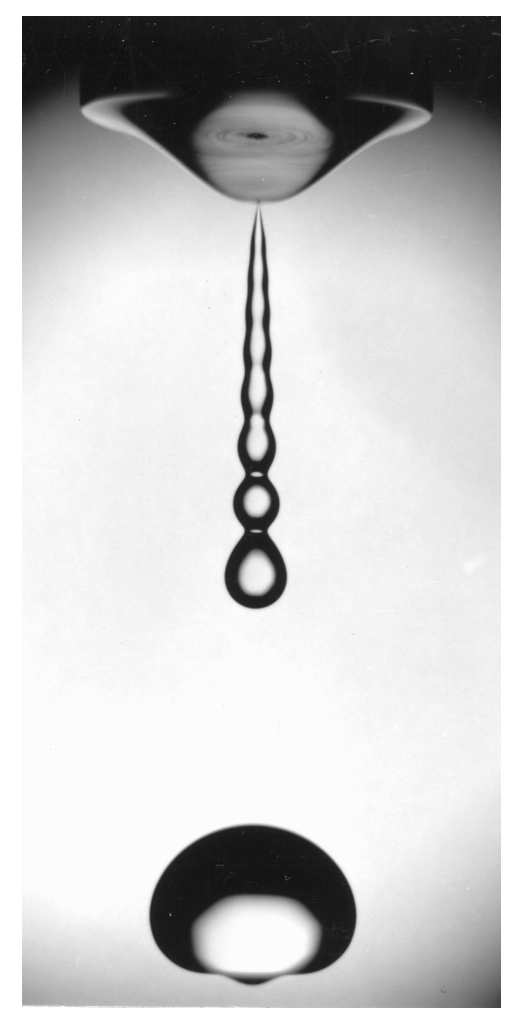

Figure 4: Water Droplet after Breakup

capillary waves cause instabilities in the primary similarity solution which themselves lead to structures that are self-similar.

Second, there is the fact that the shape of the breakup region depends upon the ratio of the viscosity of the internal fluid, $\mu_{\text {int }}$, to the viscosity of the external surrounding medium, $\mu_{\text {ext }}$. The shape of water dripping from a faucet surrounded by air (figures 3 and 4 ) in which $\mu_{\text {int }} \gg \mu_{\text {ext }}$ is different than that of a fluid falling through another fluid of approximately the same viscosity (figure 5 ) where $\mu_{\mathrm{int}} \approx \mu_{\mathrm{ext}} \cdot{ }^{13}$ Despite this both are, in fact, robust across a wide range of parameters. ${ }^{14}$ Interestingly, Sidney Nagel et al. [4] have recently demonstrated a third regime, characterized by $\mu_{\text {int }} \ll \mu_{\text {ext }}$ that fails

\footnotetext{
${ }^{13}$ Compare the shapes of the cone at the breakup in figures 3 and 5 . (Figures 3, 4, and 5 are courtesy of Sidney R. Nagel.)

${ }^{14}$ In particular, they are robust or universal over a fairly wide range of the relative viscosities of inner and outer "fluids".
} 


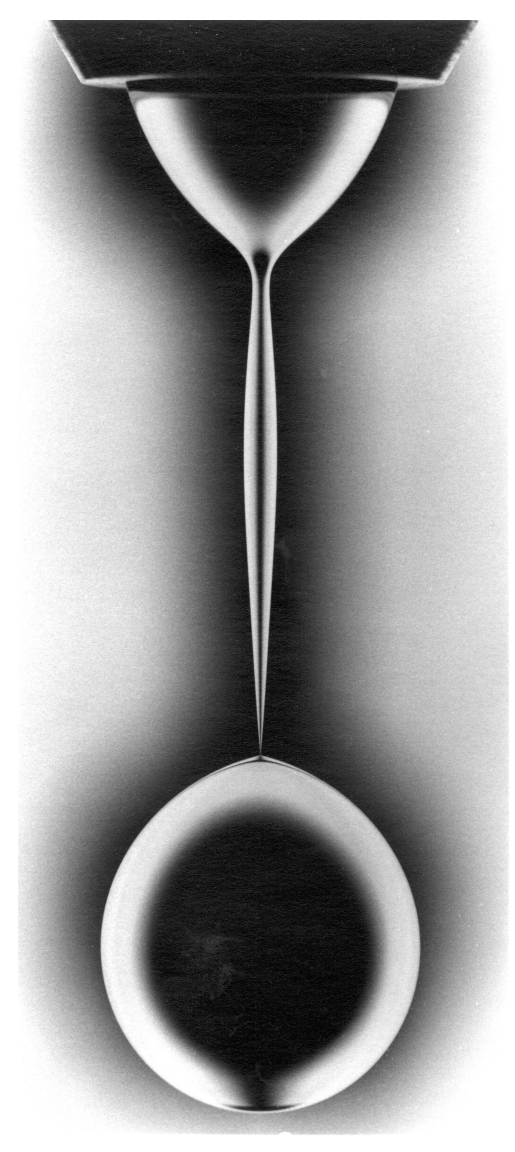

Figure 5: Two Fluid Drop Off

to exhibit universal behavior. The breakup profiles in this latter regime are nonuniversal and depend upon initial and boundary conditions in a way that the other two regimes do not.

These caveats aside, the existence of the similarity solution in the variable $\zeta$ indicates the universality of the shape of breaking droplets. For instance, compare the cone-to-sphere shape in figure 3 with the shape of the interface between the about-to-break satellite drop at the top of figure 4. This has the same cone-to-sphere shape indicating that "[t]he point of breakup would have looked the same even if it had been formed from a wave tossed into the air at the shores of Lake Michigan." [16, p. 27] Thus, the details of how the drops are formed are essentially irrelevant for their character upon breaking. 
This is a characteristic feature of universality. ${ }^{15}$

Physical discontinuities, typically are represented by singularities and divergences in their representative equations. Such singularities or divergences are often associated with the existence of scaling and, hence, are indicators of universality. We see this in the case of breaking droplets and in the case of thermodynamics at critical points. The determination of the scaling exponents (e.g. $\alpha$ and $\beta$ in equation (3)) provides a means to delimit the class of systems that exhibit the same (universal) behavior.

\section{Conclusion}

As I noted in the beginning, many physical theories are related to one another by limits that are singular - situations in which for some relevant parameter, $\epsilon$, schema (1) fails. Statistical mechanics and thermodynamics provide an interesting pair in that the limiting relationship is singular at critical points. Yet for other phases, much work has been done to demonstrate the existence of this thermodynamic limit - that is, the regularity of schema (1).

Several interesting things are going on here. First, there are two limits involved:

- The thermodynamic limit:

$$
\frac{1}{N} \rightarrow 0
$$

- The limit in which the temperature $T \rightarrow T_{c}$ from below in the case of a magnet.

If we take the thermodynamic limit first, and then let $T \rightarrow T_{c}$, we will have nonanalyticities in the statistical mechanical equations that mimic the kinks in the thermodynamic equations. On the other hand, if we let $T \rightarrow T_{c}$ first, we will not get a phase transition for any finite $N$ no matter how large. This is to say that the two limits do not commute.

Second, it is exactly at critical points (second order phase transitions) where the thermodynamic limit is singular. In other words, as we have seen, Mean Field Theory fails. The idealization becomes uncontrollable and new techniques are required to understand the subtle structures that emerge at those locations in phase. It is here that we encounter sophisticated attempts

\footnotetext{
${ }^{15}$ See [1] for an extended discussion.
} 
to deal with the singular nature of the limit. In effect, the success of these attempts provides a vindication of the universal thermodynamic representation of the phenomena. But, such a vindication is most definitely not a derivation. ${ }^{16}$

The analogy with the case of drop breakup is close indeed. The hydrodynamic equations must blow up as the breakup point is approached. Attempts must be made to see if it is possible to justify a "macroscopic" (that is, hydrodynamic) description of the physical discontinuity.

In both situations, as the singularities are approached, there is a loss of a characteristic length scale. This leads to the hypothesis of scale invariance and the idea that the large scale features of a system are virtually independent of what goes on at a microscopic level. In the thermodynamic case we see that the bulk properties of the thermodynamic systems are independent of the detailed microscopic, molecular constitution of the physical system. Similarly in the case of drop formation, the ultimate breakup profile is independent of the microscopic details of the breaking.

We ought to take very seriously the idea that real physical systems display genuine physical discontinuities - critical behavior in thermodynamic systems and finite time topological changes in the evolution of hydrodynamic free surface flows. And, we ought to take very seriously the idea that the full understanding of such physical discontinuities can be achieved only though a detailed investigation of the asymptotics of the governing equations. The appeal to asymptotics is essential to a foundationally respectable understanding of these physical discontinuities.

\footnotetext{
${ }^{16}$ It is interesting to note that in Gibbs' famous work Elementary Principles in Statistical Mechanics [9] no mention of phase transitions or critical phenomena appear despite the fact that Gibbs was an expert on the subject. The book develops, as far as possible, a reductive relationship between thermodynamics and statistical mechanics that holds away from lines of phase transition and critical points. One reason for Gibbs' caution in advocating "thermodynamic analogies" as opposed to philosopher's bridge laws connecting concepts of the two theories, was surely his recognition that such connections fail at criticality.
} 


\section{References}

[1] Robert W. Batterman. The Devil in the Details: Asymptotic Reasoning in Explanation, Reduction, and Emergence. Oxford Studies in Philosophy of Science. Oxford University Press, 2002.

[2] Michael P. Brenner, X. D. Shi, and Sidney R. Nagel. Iterated instabilities during droplet fission. Physical Review Letters, 73(25):3391-3394, 1994.

[3] Craig Callender. Taking thermodynamics too seriously. Studies in History and Philosophy of Modern Physics, 32(4):539-533, 2001.

[4] Pankaj Doshi, Itai Cohen, Wendy W. Zhang, Michael Siegel, Peter Howell andOsman A Basaran, and Sidney R. Nagel. Non-universal drop break-up. 2003.

[5] Jens Eggers. Theory of drop formation. Physics of Fluids, 7:941-953, 1994.

[6] Jens Eggers. Nonlinear dynamics and breakup of free-surface flows. Reviews of Modern Physics, 69(3):865-929, 1997.

[7] Jens Eggers. Hydrodynamic singularities. In Stefan C. Müller Jürgen Paris and Walter Zimmermann, editors, A Perspective Look at Nonlinear Media: From Physics to Biology and Social Sciences, pages 305-312, Berlin, 1998. Springer.

[8] Jens Eggers and Todd F. Dupont. Drop formation in a one-dimensional approximation of the navier-stokes equation. Journal of Fluid Mechanics, 262:205-221, 1994.

[9] J. Willard Gibbs. Elementary Principles in Statistical Mechanics: Developed with Especial Reference to The Rational Foundation of Thermodynamics. Ox Bow Press, 1981.

[10] V. L. Ginzburg. Soviet Physics, Solid State, 2:1824, 1960.

[11] Nigel Goldenfeld. Lectures on Phase Transitions and the Renormalization Group. Number 85 in Frontiers in Physics. Addison-Wesley, Reading, Massachusetts, 1992. 
[12] Dieter H. E. Gross. Microcanonical Thermodynamics: Phase Transitions in "Small" Systems. World Scientific, Singapore, 2001.

[13] Leo P. Kadanoff. Statistical Physics: Statics, Dynamics, and Renormalization. World Scientific, Singapore, 2000.

[14] Chuang Liu. Explaining the emergence of cooperative phenomena. In Philosophy of Science 66 (Proceedings), pages S92-S106, 1999.

[15] Ernest Nagel. The Structure of Science: Problems in the Logic of Scientific Explanation. Harcourt, Brace, \& World, 1961.

[16] Sidney R. Nagel. Shadows and ephemera. Critical Inquiry, 28(1):23-39, Autumn 2001.

[17] Thomas Nickles. Two concepts of intertheoretic reduction. The Journal of Philosophy, 70(7):181-201, 1973.

[18] Kenneth Schaffner. Reductionism in biology: Prospects and problems. In et al. R. S. Cohen, editor, PSA 1974, pages 613-632. Philosophy of Science Association, D. Reidel Publishing Company, 1976.

[19] X. D. Shi, Michael P. Brenner, and Sidney R. Nagel. A cascade structure in a drop falling from a faucet. Science, 265(5169):219-222, 1994.

[20] Lawrence Sklar. Types of inter-theoretic reduction. The British Journal for the Philosophy of Science, 18:109-124, 1967.

[21] Lawrence Sklar. Physics and Chance: Philosophical Issues in the Foundations of Statstical Mechanics. Cambridge University Press, Cambridge, 1993.

[22] Lawrence Sklar. Theory and Truth: Philosophical Critique within Foundational Science. Oxford University Press, Oxford, 2000. 